\title{
DIREITOS HUMANOS À ÁGUA E AO ESGOTAMENTO SANITÁRIO EM CONTEXTOS ONDE AS DEMANDAS INDIVIDUAIS PREVALECEM
}

\section{HUMAN RIGHTS TO WATER AND SANITATION IN RURAL CONTEXT WHERE INDIVIDUAL DEMANDS PREVAIL}

\author{
Washington Lima dos Santos ${ }^{(1)}$ \\ Engenheiro Químico, mestre em Engenharia Química e mestrando pelo programa em Saneamento, Meio Ambiente e \\ Recursos Hídricos SMARH (UFMG). \\ Naiara Ramos de Oliveira ${ }^{(2)}$ \\ Engenheira Civil e mestranda pelo programa SMARH (UFMG). \\ Hebert Medeiros Gontijo ${ }^{(3)}$ \\ Engenheiro Mecânico, mestre em Desenvolvimento Sustentável e doutorando pelo programa SMARH (UFMG). \\ Sonaly Cristina Rezende Borges de Lima ${ }^{(4)}$ \\ Engenheira Civil, mestre pelo SMARH (UFMG), doutora pelo Centro de Desenvolvimento e Planejamento Regional da \\ UFMG e pós-Doutora pela UFMG. Atual docente do departamento de Engenharia Sanitária e Ambiental da UFMG.
}

\section{E-mail ${ }^{(1)}$ : wwashingtonn@hotmail.com}

\section{RESUMO}

A relatoria especial da ONU para o direito humano à água potável e ao esgotamento sanitário tem produzido um aporte teórico e guia importante. Em convergência, no Brasil, a política de saneamento tem apresentado avanços significativos, como exemplo, com o lançamento do Programa Nacional de Saneamento Rural (PNSR). O objetivo deste artigo é apresentar princípios (igualdade e não discriminação; e responsabilização) e requisitos (aceitabilidade, acessibilidade, qualidade e segurança) para a garantia dos DHAS, abordados nos relatórios da ONU, e confrontá-los aos desafios da ruralidade caracterizada pela predominância de soluções individuais e de pequena escala. Conclui-se que o momento de discussão é oportuno e que as soluções individuais são pautas sensíveis aos princípios e padrões dos DHAS. Por fim, entende-se que discutir a elevação do nível de provisão de serviços do setor é compreender que um nível mais alto dos serviços significa um melhor atendimento aos princípios e padrões de DHAS.

\begin{abstract}
The UN Special Rapporteur on the human right to drinking water and sanitation sewerage has produced an important theoretical contribution and guide. In convergence, in Brazil, the sanitation policy has shown significant advances, for example, the launch of the National Rural Sanitation Program (PNSR). The purpose of this article is to present principles (equality and non-discrimination; and accountability) and requirements (acceptability, acceptability, quality and safety) to guarantee HRWS, addressed in UN reports, and confront them with the challenges of rurality characterized by the predominance of solutions individual and small scale. It is concluded that the time for discussion is opportune and that individual solutions are sensitive to the principles and criteria of the HRWS. Finally, it is understood that discussing the increase in the level of provision of services in the sector is to understand that a higher level of services means better compliance with HRWS standards.
\end{abstract}

Palavras-chave: Direitos Humanos. Água e saneamento. Áreas Rurais. Soluções Individuais. Pequena Escala.

Key words: Human rights. Water and sanitation. Rural Areas. Individual Solutions. Small scale.

\section{INTRODUÇÃO}

A Assembleia Geral das Nações Unidas declarou o acesso à água limpa e esgotamento sanitário como direitos humanos essenciais "para gozar plenamente a vida e todos os outros direitos humanos" 
(A/RES/64/292, 2010). Posteriormente, em 2013, a Resolução 24/18 do Conselho de Direitos Humanos reconheceu que tal direito permite que todas as pessoas tenham acesso a água em quantidade suficiente, segura, aceitável, física e economicamente acessível para uso pessoal e doméstico. Ademais, prevê a presença de instalações hidrossanitárias seguras, higiênicas, confiáveis e aceitáveis, capazes de proporcionar privacidade e assegurar a dignidade (ONU, 2013).

Desde então, relatórios de distintas instituições internacionais (ONU, OMS, UNICEF, UNESCO etc.), sobre os mais variados aspectos, têm sido produzidos e vêm acompanhando as tendências, urgências, escolhas e desdobramentos referentes à declaração dos Direitos Humanos à Água Potável e ao Esgotamento Sanitário (DHAES). Não obstante, uma relatoria especial para o direito humano à água potável e ao esgotamento sanitário tem produzido um aporte teórico e guia importante para o campo político, técnico, acadêmico e para o empoderamento da população, em geral.

Este artigo possui como objetivo apresentar princípios e requisitos para a garantia dos direitos humanos à água e ao esgotamento sanitário, abordados nos relatórios da ONU, e confrontá-los aos desafios da ruralidade caracterizada pela predominância de soluções individuais e de pequena escala.

\section{METODOLOGIA}

O estudo abrange a organização de conteúdos de documentos oficiais provenientes da ONU, em sua relatoria especial voltada aos direitos humanos à água e ao saneamento: A/70/203, A/71/302, A/HRC/30/39, A/HRC/33/49 e A/HRC/42/47; e da Funasa/UFMG, do documento central do PNSR e dos tomos específicos sobre Ruralidade e Matrizes tecnológicas presentes na Série Subsídios ao Saneamento Rural.

A partir de uma breve apresentação do PNSR e do delineamento da ruralidade em questão (secção 3.1), reconhecida pela predominância das soluções individuais ou de pequena escala, foram escolhidos dois temas caracterizados pelos princípios dos DHAES, associados a subtemas representados por critérios dos DHAES (secção 3.2).

O primeiro tema é pautado no princípio da igualdade e não discriminação e a partir dele é explorado o critério da aceitabilidade. O segundo tema é pautado no princípio da responsabilização e suas relações com a disponibilidade dos serviços; a qualidade e segurança da água e sua quantidade.

\section{RESULTADOS E DISCUSSÃO}

\section{1 - Perspectivas do Programa Nacional de Saneamento Rural (PNSR)}

No Brasil, a política de saneamento teve nas duas últimas décadas avanços significativos que emergiram da crítica aos processos construídos, que resultaram na manutenção das desigualdades sociais. O Plano Nacional de Saneamento Básico (Plansab), lançado em 2013, ampliou as possibilidades para o saneamento se desenvolver sustentado por medidas estruturais e estruturantes, repercutindo a importância dos princípios e critérios dos DHAES. O Plansab instituiu três programas para a realização das diretrizes propostas para o alcance das metas, em um horizonte de 20 anos: Saneamento Integrado, Saneamento Estruturante e Saneamento Rural, sendo este último de responsabilidade do Ministério da Saúde, ao contrário dos dois primeiros, que ficaram com o Ministério das Cidades e hoje se encontram no Ministério do Desenvolvimento Regional.

O Programa Saneamento Brasil Rural foi lançado em dezembro de 2019, trazendo aportes relevantes para o setor. O Programa foi construído em um contexto de discussões amplo, rico e abrangente, contando com a participação dos governos e da sociedade civil organizada. Durante sua formulação, a equipe da UFMG e colaboradores elaboraram um material que abarca aspectos que subsidiaram o Programa e que reunem a sua memória, e que tem contribuído para estudos e projetos voltados às 
áreas rurais. O intenso debate que se constituiu no processo de formulação do Programa Saneamento Rural, apesar de ter sido abafado no atual contexto político e pandêmico, deixou muitos frutos, além do rico material bibliográfico, alianças se formaram para a defesa do saneamento. Esse contexto de avanços significativos e atualizações controversas resultou na Lei $n^{\circ} 14.026 / 2020$, que muda a Lei $n^{\circ}$ 11.445/2007 e dita novos rumos para a Política Nacional de Saneamento, em meio à ambiguidade associada às novas formas de gestão dos serviços. O desafio contemporâneo envolve a compreensão das ruralidades e respectivas demandas de saneamento básico, em meio a orientações privatistas que afetam a organização dos serviços públicos, tendo em vista a obrigatoriedade da licitação pública de contratos de saneamento e de projetos de regionalização para a gestão dos serviços, estabelecidos na nova Lei.

O estudo para a formulação do pnsr abarcou uma ampla revisão de base conceitual e metodológica que visa orientar ações nos municípios, tendo como referência suas ruralidades. Além de aportes teóricos, que revelaram a complexidade do rural e a importância de interpretá-lo a partir de suas especificidades, também houve a definição de uma tipologia que agregou setores censitários do IBGE, conforme critérios de densidade demográfica e circunvizinhança, articulando a cada ruralidade as prováveis soluções sanitárias, no plano da tecnologia, gestão, educação e participação social (BRASIL,2019). Os setores censitários foram agrupados segundo a premissa de que as ações individuais de saneamento se destinam a domicílios localizados em áreas de ocupação remota, dispersos no território e distantes uns dos outros. As ações coletivas de saneamento, por sua vez, se aplicam a domicílios distribuídos no território em diferentes escalas de aglomeração e de proximidade com as áreas urbanas.

Este estudo foca nas ruralidades associadas aos setores censitários que possuem práticas individuais consolidadas, representadas pelas aglomerações menos adensadas isoladas (que possuem setores censitários de códigos 5, 6 e 7) e em áreas sem aglomerações, com domicílios relativamente próximos de aglomerações ou isolados (setores censitários de código 8). Em linhas gerais, a ocupação desses lugares revela maiores distâncias entre as residências ou entre pequenas aglomerações de domicílios. As práticas individuais prevalecem apesar de coexistirem com soluções coletivas de gestão incipiente.

O PNSR prioriza o atendimento de todas as demandas municipais urbanas e rurais, sempre que possível, por serviços públicos constituídos. Mas, considera como importante estratégia para o atendimento das distintas demandas a manutenção da prática vigente, quando necessário, com adequação técnica. Para isso, o PNSR define três eixos estratégicos para o saneamento rural, trazendo a educação e participação social como um deles. A universalização do saneamento rural depende da participação ativa dos interessados desde a fase de planejamento, concepção, execução, manutenção e avaliação das atividades realizadas (BRASIL, 2019).

Segundo o relatório A/70/203 (2015), as estruturas necessárias para suprir este déficit devem ser implantadas a fim de garantir a disponibilidade de serviços, programas definidos em políticas, instituições e pessoal suficiente, capazes de construir, manter e gerenciar os serviços. Neste sentido, o PNSR traz uma matriz tecnológica alinhada às particularidades locais e instrumentos que envolvem a gestão e operação dos serviços, por técnicos e usuários (BRASIL, 2019). Propõe que a gestão dos serviços de saneamento se dê por processos multiescalares, com o envolvimento de distintos atores sociais, de diferentes esferas ou escalas de atuação. Um envolvimento dinâmico entre a população e o poder público que dispõe dos recursos necessários para promover ações que garantam o atendimento com regularidade (BRASIL, 2019).

Uma preocupação transparece nos relatórios no que tange ao envolvimento do setor privado, dificultando a disponibilidade dos serviços para as áreas rurais, que oferecem poucas possibilidades de retorno financeiro. Frequentemente, o setor privado, apoiado pelo contratante, restringe seus contratos de atuação, excluindo as áreas rurais e estas seguem sob responsabilidade pública, não atingindo prioridade necessária para contar com recursos humanos e econômicos (A/75/208, 2020). 


\section{2 - DHAS em contextos de demandas individuais}

O acúmulo teórico e das experiências práticas oriundo do debate acerca dos direitos humanos tem contribuído amplamente para o planejamento de ações que resultam em saúde e qualidade de vida. A Relatoria A/70/203, por exemplo, resulta de uma discussão introduzida pela Resolução 24/18, que repercutiu no trabalho do Comitê de Direitos Econômicos, Sociais e Culturais (E/C.12/2002/11 e $\mathrm{A} / \mathrm{HRC} / 12 / 24)$ e nas relatoria especial (A/HRC/12/24). Esta Relatoria revisa e esclarece o significado dos padrões e princípios de direitos humanos à água, esgotamento sanitário e higiene (WASH: water, sanitation, hygiene). Abrange princípios que correspondem ao alcance de condições de não discriminação e igualdade substantiva, participação social, acesso à informação, responsabilização e sustentabilidade. E abrange padrões voltados à disponibilidade de água e esgotamento sanitário, à acessibilidade física à água e ao banheiro, à segurança e qualidade da água distribuída, à acessibilidade econômica e à aceitabilidade da água e da solução para o esgotamento sanitário, bem como a higiene/limpeza, considerando, nesse particular, as condições de privacidade e dignidade humana. Os princípios e padrões dos DHAES não são associados a padrões fixos. Ao contrário, denotam as peculiaridades de cada demanda. No entanto, as normativas fornecem um quadro que pode apoiar os Estados e as agências internacionais na busca de serviços com caráter mais prescritivo, pautados em regras e instruções (A/70/203, 2015).

Os DHAES estão pautados no princípio da igualdade e não discriminação, o que leva à necessidade de políticas que priorizem os "setores vulneráveis" da população rural para o alcance da universalidade (BRASIL, 2019). A aceitabilidade possui forte relação com o princípio de igualdade e não discriminação, tendo em vista que as diferenças socioculturais e econômicas existentes nas distintas ruralidades repercutem nos distintos modos de vida e, consequentemente, de uso das técnicas empregadas no abastecimento de água e no esgotamento sanitário. Para determinar o tipo de tecnologia a ser projetada, construída e mantida em conformidade com as demandas a aceitabilidade ganha papel central. O PNSR atribui significativa importância à aceitabilidade no atendimento às populações rurais e sua estreita ligação com os padrões culturais e sociais estabelecidos. A apropriação/assimilação da técnica e sua adaptação ao cotidiano minimiza o risco de não ser utilizada ou incorporada aos costumes locais (BRASIL, 2019).

A aceitabilidade depende de uma série de fatores e pode ser alcançada levando-se em consideração os hábitos, as preferências, as necessidades e as crenças dos usuários. As mudanças necessárias à introdução de uma nova solução sanitária também decorrem da participação social e da educação popular, que envolve a troca de saberes e considera o contexto de aprendizagem. Atividades para a promoção de debates e reflexões são de grande importância para incentivar a mudança de comportamento e levar a uma maior aceitabilidade dos serviços (A /71/302, 2016).

Os princícpios da acessibilidade e da aceitabilidade têm ampla conexão, por exemplo, na medida que a necessidade de uso de múltiplas fontes, em condições adversas de acesso físico e financeiro, leva a padrões de aceitação mais baixos, em termos de quantidade e qualidade. Soluções adequadas não comprometem a saúde e não interferem na garantia de outros serviços essencias (A/HRC/45/10,2020). Quando não há garantia de acessibilidade física à água e banheiro, há comprometimento da saúde da população e sobrecarga para as mulheres, que despendem maior tempo e energia para realizar a gestão da água no domicílio (ONU, 2016).

O princípio da responsabilização também é de grande interesse para as discussões envolvendo o saneamento em ruralidades nas quais predominam soluções individuais. Esse princípio serve como pano de fundo para a discussão de padrões dos DHAES relativos à disponibilidade dos serviços, à qualidade e segurança e quantidade de água. Cabe ao titular, o ente municipal, garantir o atendimento da população com soluções adequadas de saneamento, mas, via de regra, os prestadores de serviço 
focam atenção às áreas urbanizadas e o município assume - de forma precária e incipiente, na maioria das vezes, $-\mathrm{o}$ atendimento às demandas rurais reconhecidas. Isso porque na ruralidade aqui tratada, o usuário costuma ser o seu próprio "prestador de serviço", a depender da proximidade de áreas urbanizadas, nessas áreas estão presentes serviços informais e/ou auto regulados, tanto para uso domiciliar, quanto para ambientes públicos.

No quesito qualidade da água, os relatórios da ONU abordam o padrão de qualidade relacionado ao uso humano (A/70/203, 2015; A/HRC/36/45, 2017; A/HRC/45/10, 2020). Tecnicamente, a OMS e os Estados devem publicar diretrizes com a presença de limites para uma ampla variação de substâncias. Trata-se de aspecto sensível diante das ruralidades, com suas múltiplas possibilidades de captação e respectivas exigências em termos de segurança e qualidade, demandando aporte tecnológico para avaliação de contaminantes. A caracterização da qualidade exige ainda periodicidade de avaliação, tanto nos domicílios quanto nos espaços públicos.

Vale destacar que os objetivos 2030 da ONU e as novas abordagens internacionais exigem discussão com maior profundidade sobre o padrão de qualidade da água no tocante ao esgotamento sanitário, para o reuso e/ou descarte das águas residuárias. Assim, ainda que os relatórios abordem especificamente o direito à água potável, é crucial a discussão integrada no âmbito dos recursos hídricos. A qualidade, em relação ao esgotamento sanitário, refere-se à higiene e segurança das instalações sanitárias, tanto para o uso quanto para a manutenção e facilidade de limpeza. Deve impedir o contato de seres humanos e animais, incluindo insetos, com excrementos humanos a fim de evitar a disseminação de doenças (A/70/203, 2015). Essas realidades rurais são marcadas pela defecação a céu aberto e por soluções individuais autogestionadas.

Outro padrão para o cumprimento dos DHAES é o da disponibilidade, que se refere à quantidade suficiente de água para o atendimento às demandas existentes e a continuidade/regularidade desse atendimento. No Brasil a diversidade de biomas e consequentemente, a variação em termos de disponibilidade hídrica resulta em situações muito distintas de atendimento em áreas rurais: variando do atendimento por sistemas coletivos com níveis distintos de precariedade até as soluções individuais canalizadas no domicílio ou terreno, ou, ainda, as soluções caracterizadas pela coleta manual de água em poços rasos e mananciais superficiais, muitas vezes longe das residências, e transportada em baldes e latas até o domicílio (BRASIL, 2019).

\section{CONCLUSÕES}

Ampliar o acesso à água e ao esgotamento sanitário no Brasil é um dever do Estado e as normativas dos DHAES têm conduzido a discussão dos meios de se alcançar a universalidade, com equidade e integralidade. Nas áreas rurais, onde é indispensável o envolvimento dos usuários para que as demandas sejam interpretadas e atendidas, as práticas sanitárias são caracterizadas pela ausência da cultura de atuação do poder público, sobressaindo-se a cultura e o saber local, influenciados pelos contextos ambiental e demográfico. O reconhecimento desta premissa deve nortear as decisões acerca da gestão instituída, para apropriação e uso adequado da técnica. Além disso, educação e participação social são essenciais ao alcance da efetividade da gestão em saneamento rural e para a adoção de soluções perenes e sustentáveis (BRASIL, 2019).

Abordar princípios e padrões de DHAES é discutir a elevação do nível de provisão de serviços de água e esgotamento sanitário, onde um nível mais alto dos serviços significa um melhor atendimento aos padrões de direito humanos. A partir do reconhecimento de vários critérios (A/HRC/45/10, 2020), existe um quadro dos ODSs em que "escadas" adotadas pelo Programa Conjunto de Monitoramento do Abastecimento de Água, Esgotamento Sanitário e Higiene, da OMS e UNICEF, revelam 
possibilidade de avaliar níveis mais próximos do esperado em DH. Essa questão reforça a importância de se planejar ações que sejam o mais adaptadas possível às distintas ruralidades, considerando o atendimento digno e a realização dos $\mathrm{DH}$, a partir da conjugação de esforços do poder público e da sociedade civil organizada.

\section{AGRADECIMENTOS}

Os autores agradecem às agências de fomento CAPES e CNPq pelas bolsas concedidas.

\section{REFERÊNCIAS}

BRASIL. Ministério da Saúde. Fundação Nacional de Saúde. Programa Nacional de Saneamento Rural. Brasília: Funasa, 2019. Disponível em: http://www.funasa.gov.br:8080/documents/20182/ 38564/MNL_PNSR_2019.pdf/08d94216-fb09-468e-ac98-afb4ed0483eb. Acesso em: 06 Out. 2021.

Lei 14026, de 15 de julho de 2020. Atualiza o Marco Legal de Saneamento Básico. Disponível em: https://presrepublica.jusbrasil.com.br/legislacao/875819060/lei-14026-20. Acesso em: 01 Out. 2021.

ORGANIZATION OF THE UNITED NATIONS (UN). HUMAN RIGHTS COUNCIL. Promotion and protection of all human rights, civil, political, economic, social and cultural rights, including the right to development: Report of the Special Rapporteur on the human right to safe drinking water and sanitation (A/HRC/33/49). UN, 2016. Disponível em: https://documents-ddsny.un.org/doc/UNDOC/GEN/G16/166/97/PDF/G1616697.pdf?OpenElement. Acesso em: 01 Out. 2021.

- Human rights questions, including alternative approaches for improving the effective enjoyment of human rights and fundamental freedoms: Human right to safe drinking water and sanitation - Note by the Secretary-General (A/70/203). UN, 2015. Disponível em: https://undocs.org/A/70/203. Acesso em: 01 Out. 2021.

. Human rights to water and sanitation in spheres of life beyond the household with an emphasis on public spaces - Report of the Special Rapporteur on the human rights to safe drinking water and sanitation (A/HRC/42/47). UN, 2019.2 Disponível em: https://ap.ohchr.org/documents/dpage_e.aspx?si=A/HRC/42/47. Acesso em: 01 Out. 2021.

- Progressive realization of the human rights to water and sanitation: report of the Special Rapporteur on the Human Rights to Safe Drinking Water and Sanitation (A/HRC/45/10). UN, 2020. Disponível em: https://digitallibrary.un.org/record/3875004\#record-files-collapse-header. Acesso em: 01 Out. 2021.

. Promotion and protection of all human rights, civil, political, economic, social and cultural rights, including the right to development: Report of the Special Rapporteur on the human rights to safe drinking water and sanitation: note by the secretariat (A/HRC/39/55). UN, 2018. Disponível em: https://undocs.org/pdf?symbol=en/A/HRC/39/55. Acesso em: 01 Out. 2021.

Promotion and protection of all human rights, civil, political, economic, social and cultural rights, including the right to development (A/HRC/RES/24/18). UN, 2013. Disponível em: https://undocs.org/A/HRC/RES/24/18. Acesso em: 01 Out. 2021.

. Promotion and protection of all human rights, civil, political, economic, social and cultural rights, including the right to development: Report of the Special Rapporteur on the human rights to safe drinking water and sanitation - Note by the Secretariat (A/HRC/36/45). UN, 2017. Disponível em: https://undocs.org/A/HRC/36/45. Acesso em: 01 Out. 2021. 
Promotion and protection of human rights: human rights questions, including alternative approaches for improving the effective enjoyment of human rights and fundamental freedoms: Human rights and the privatization of water and sanitation services -Note by the Secretary-General (A/75/208). UN, 2020. Disponível em: https://undocs.org/en/A/75/208. Acesso em: 01 Out. 2021.

Promotion and protection of human rights: human rights questions, including alternative approaches for improving the effective enjoyment of human rights and fundamental freedoms: Human right to safe drinking water and sanitation - Note by the Secretary-General (A/71/302). UN, 2016. Disponível em: https://undocs.org/A/71/302. Acesso em: 01 Out. 2021.

Resolution adopted by the General Assembly on 28 July 2010 (A/RES/64/292). UN, 2010. Disponível em: https://undocs.org/A/RES/64/292. Acesso em: 01 Out. 2021. 